\title{
Development of the acinus in the human lung
}

\author{
ALISON HISLOP and LYNNE REID \\ Department of Experimental Pathology, Cardiothoracic Institute, Brompton Hospital, London SW3
}

\begin{abstract}
Hislop, Alison, and Reid, Lynne (1974). Thorax, 29, 90-94. Development of the acinus in the human lung. Development and remodelling of the acinus (those structures distal to the terminal bronchiolus) occurs during fetal life and childhood. Multiplication of the acinar air spaces and the structural changes they undergo have been described and represented schematically by summarizing previous studies, particularly those of the last two decades.

Most acinar airways are present before birth. Future respiratory bronchioli are represented by the 19th intrauterine week; alveolar ducts are present as saccules by the 28th week. Alveolar sacs and alveoli, as properly described, do not appear until after birth: alveoli increase in number, particularly in the first seven or so years of life, and in size with thoracic growth.
\end{abstract}

During the last two decades there has been much work on lung development and, in particular by Professor E. A. Boyden, on the development of the structures within the acinus. It was he who suggested that it would be useful to collate the findings at various ages and to present them in a comprehensive but simplified way. In the present report acinar growth has been summarized schematically, both the time of appearance of the constituent structures and the remodelling that they undergo from the early months of gestation through childhood. It has been our aim to give a general statement of the development of the acinus rather than the detail of the particular cases upon which the work is based.

\section{MATERIAL AND METHODS}

Over the last 10 years Professor Boyden has used the Born waxplate method to reconstruct the acinus at various ages ranging from 30 weeks' gestation to $6 \frac{2}{3}$ years (Boyden, 1965, 1967, 1969, 1971 ; Boyden and Tompsett, 1965). These models have been concerned mainly with airways and alveoli. In this department serial and step sections of the pre- and intra-acinar regions in adults, children, and fetuses (Reid and Rubino, 1959; Bucher and Reid, 1961 ; Elliott and Reid, 1965; Hislop and Reid, 1972, 1973a and b) have been used to study the development of airways, arteries, and veins by assessment of the length and number of branches and the measurement of acinar length. These results have been particularly important in deciding the pattern

Requests for reprints: Professor Lynne Reid, Department of Experimental Pathology, Cardiothoracic Institute, Brompton Hospital, London SW3 of antenatal growth. Quantitative analysis using random sampling techniques has been applied to the growing lung, and the number and shape of alveoli have been estimated (Dunnill, 1962; Davies and Reid, 1970) and also size (Davies and Reid, 1970).

The composite diagram shown in the Figure is based on the results of a number of studies. It represents what happens to the terminal airways and alveoli from the antenatal period through to the adult. Certain ages have been selected for illustration in the diagrams either because they represent landmarks or because serial reconstructions are available. Each of the stages is described in detail as well as the changes that occur between. In the diagram each airway branch or generation is shown as the same length, both at all ages and at all levels along the airways. Thus increase in length of the pathway in the diagram represents an increase in the number of generations. It also has the advantage that a given generation can be traced through all ages. The actual increase in size is shown by the measurement of an acinus at the given age. The acinus shown is not a reproduction of a particular acinus but a representation of the characteristic features of growth.

\section{DEFINITIONS}

The names used to describe the stages of fetal lung development are those suggested by the Commission on Embryological Terminology (1970):

1. Embryonic period-lasts for five weeks after ovulation (Boyden (personal communication) prefers to extend this to the 6th week when segmental airways appear

2. Pseudoglandular period-extends from the 5 th week until the 17 th week of gestation 


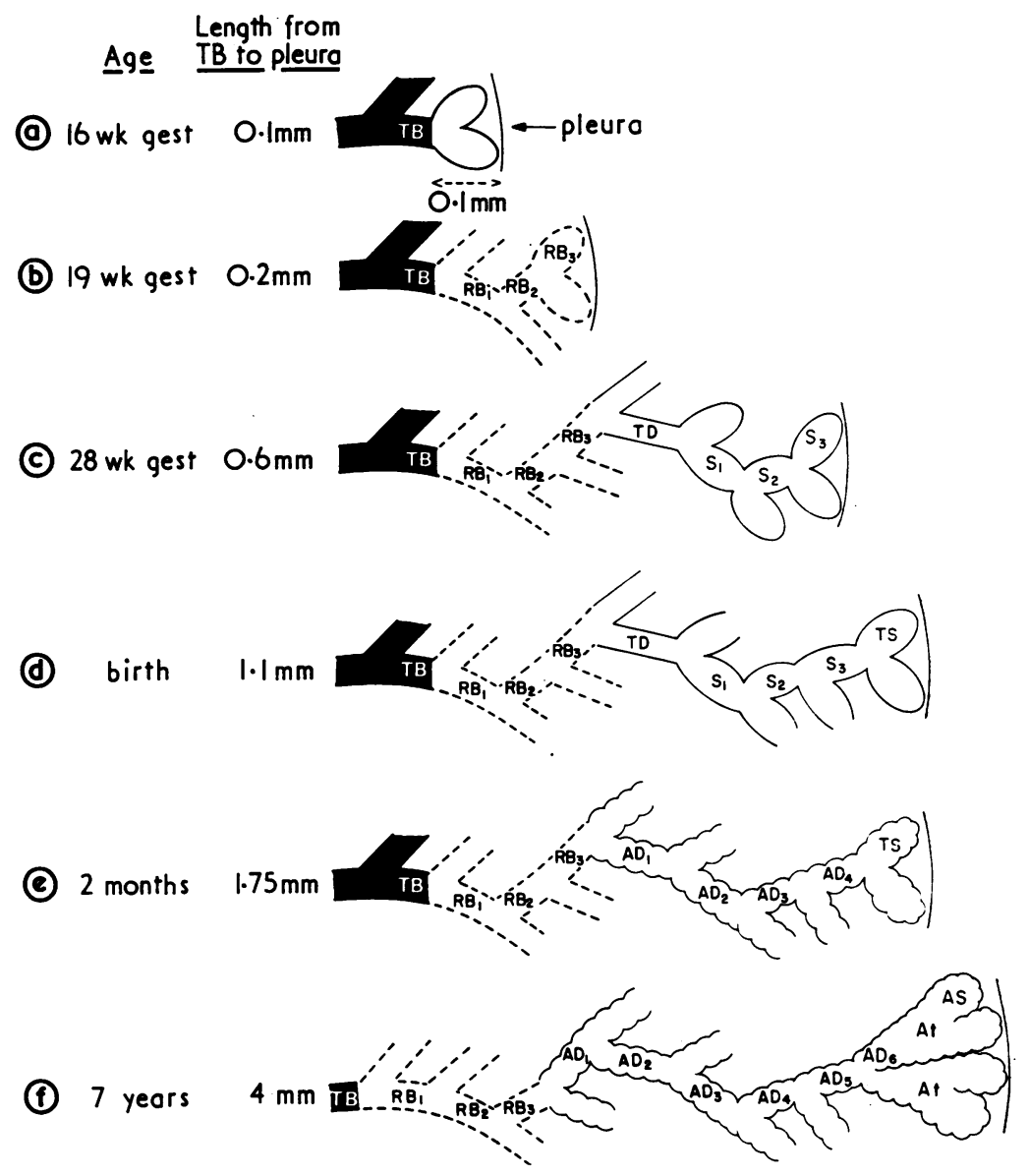

FIGURE (a tof). Diagrammatic representation of the acinus at six stages of development. At all ages airway generations are drawn the same length, so that increase in length represents an increase in generations. A given generation may be traced down the same vertical line, permitting remodelling in its structure to be followed. Actual increase in size is shown by the length from terminal bronchiolus $(T B)$ to pleura. $R B$, respiratory bronchiolus; $T D$, transitional duct; $S$, saccule; $T S$, terminal saccule; $A D$, alveolar duct; $A t$, atrium; $A S$, alveolar sac.

3. Canalicular period-has been suggested to last from the 13 th to the 25 th week by Boyden (1972a, b), while Loosli and Potter (1951) have suggested from the 16 th to the 24 th week

4. Terminal sac period-this lasts from the end of the canalicular period until term; the name has superseded 'alveolar', suggested by Loosli and Potter (1951)

The structural limits within which the anatomical terms used in this paper have been applied are given below:

Terminal bronchiolus-the last airway generation along any pathway before a respiratory bronchiolus is reached: it is lined by ciliated epithelium and supplies an acinus.
Respiratory bronchiolus-in the adult, alveoli open into the lumen of this type of airway with part of the wall lined by ciliated epithelium. During early development the future alveolar part is lined by flattened epithelium.

Saccule-this structure is seen only in the three months before and at least two months after birth and is a large, thin-walled air space simple in outline and lined by flattened epithelium. It is transformed into an alveolar duct.

Alveolar duct-in the adult this has alveoli opening into it and nowhere cuboidal epithelium. The electron microscope demonstrates that this structure is lined by alveolar epithelial cells. Muscle and elastic fibres are present in the mouths of the alveoli. 
Atrium-the name given by Miller (1937) to the distal part of the last alveolar duct beyond the level where muscle disappears. An atrium usually gives rise to at least two alveolar sacs.

Alveolar sac-the structure seen in the adult from which only alveoli arise; it is the ultimate airway.

\section{RESULTS}

INTRA-UTERINE DEVELOPMENT During the embryonic period, at the end of the fourth week a ventral diverticulum arises from the lower end of the tracheo-oesophageal sulcus; it is lined by endodermal epithelium and invested by splanchnic mesenchyme. This diverticulum bifurcates to form the two lung sacs; each gives rise to three lobar buds. By the end of the sixth week all bronchopulmonary segments have appeared. Further branching of the airways into the mesenchyme forms the bronchial tree. In section, the lung has a 'pseudoglandular' appearance that lasts up to 16 weeks' gestation.

At 16 weeks' gestation (Figure a) The airways are blind tubules lined by columnar or cuboidal epithelium. All pre-acinar airway branching is complete (Bucher and Reid, 1961) and since the penultimate generation is the prospective terminal bronchiolus, only one airway generation is found within the acinus which measures about $0.1 \mathrm{~mm}$ in axial length (that is, from the end of the terminal bronchiolus to the distal edge of the acinus).

After 16 weeks' gestation, and during the canalicular period, new generations grow. The epithelium of these and the distal airways becomes so thin in places that it is only with the electron microscope that it is apparent as a continuous layer. This flattened epithelium forms the alveolar epithelium of the adult (Campiche, Gautier, Hernandez, and Reymond, 1963; Meyrick and Reid, 1970). These airways are future 'respiratory bronchioli'. There is growth of capillaries adjacent to the respiratory bronchioli and the region becomes vascularized.

At 19 weeks' gestation (Figure b) The last generation that was present at 16 weeks now has a thinned wall and there are two or so additional generations of respiratory bronchioli. The number of respiratory bronchioli in each acinus at this age varies but the acinus now measures about $0.2 \mathrm{~mm}$ along its axial length. (One lateral pathway at 20 weeks' gestation was described by Palmer (1936).)

During the following weeks there is further branching and remodelling of intra-acinar airways. At 24 weeks' gestation the lung enters the terminal sac period characterized by the appearance of terminal clusters of airways termed 'saccules'.

At 28 weeks' gestation (Figure c, based on Boyden (1971)) Three generations of respiratory bronchioli are shown, though this number may vary. Their lining at this age includes both cuboidal and, in parts, flat epithelium. Distal to these are one or two generations of transitional ducts that are short, straight airways lined by flattened epithelium (Boyden, 1965). From each, several generations of saccules arise, each so short that the appearance is of an irregular cluster of air spaces. These saccules are large, thin-walled, irregular in shape, lined by flattened epithelium. and separated by a loose connective tissue matrix (Boyden, 1969). They have a 'blood/gas barrier' of the same thickness as that of the adult alveoli (Lauweryns and Rosan, 1971) and, from this age, suffice for respiration.

From 28 weeks' gestation to birth there is lengthening of saccules with perhaps growth of additional generations, the last being called a 'terminal saccule'.

At birth (Figure d, based on Boyden and Tompsett (1965)) Three generations of respiratory bronchioli, one of transitional duct and three of saccules, ending in a terminal saccule are shown, but in different acini more or fewer may be identified. By this time the respiratory bronchioli are actually increased in length. The saccules have shallow depressions in their walls which increase the surface area and can only be considered primitive alveoli (Boyden and Tompsett, 1965) since they do not have the typical cup shape of the adult alveolus. The acinus is about $1 \cdot 1 \mathrm{~mm}$ along its axial length.

At term the 'blood/gas barrier' is the same thickness as in the adult (Lauweryns and Rosan, 1971) although the alveolar walls appear thicker owing to a larger amount of connective tissue between capillaries. Elastic fibres are confined to the mouth of the alveolar opening while, in the adult, they are found throughout the alveolar walls. This arrangement may facilitate expansion during postnatal development (Loosli and Potter, 1959).

POST-Natal DEVElopment Between the sixth and eighth postnatal week (Boyden, 1965; Boyden and Tompsett, 1965) true alveoli rapidly develop. The respiratory bronchioli elongate and alveoli 
bulge from the areas of flattened epithelium. Saccules and transitional ducts are converted into alveolar ducts by their lengthening and by the deepening of the primitive alveoli in their walls.

At 2 months (Figure e, based on Boyden and Tompsett (1965)) The representative acinus has three generations of respiratory bronchioli and four of alveolar ducts that represent remodelled saccules; a terminal saccule is still present. All these are lined by alveoli (Boyden, 1965 ; Boyden and Tompsett, 1965). The acinus is $1.75 \mathrm{~mm}$ along its axial length.

During childhood, as each part of the acinus increases in length, there is rapid multiplication of alveoli. Reconstruction would seem to indicate that increase in number is mainly due to development of new alveoli along alveolar ducts. After birth there is little further branching but each terminal saccule produces, probably by budding, a number of alveolar sacs (one to four) within the same generation, each sac containing many alveoli. The proximal part of the terminal saccule becomes an alveolar duct, the distal part the atrium-the name given to this airway beyond the last muscle in its walls.

Remodelling of the airways also occurs and may be of several types:

(1) along some pathways the terminal bronchiolus may be transformed into an extra generation of respiratory bronchioli by 'centripetal alveolization' (Boyden and Tompsett, 1965) ;

(2) distal respiratory bronchioli may be transformed into alveolar ducts by increasing alveolization of their wall ;

(3) further branching of alveolar ducts may give rise to another one or two generations ; or

(4) an increase in length of all generations may occur without any increase in their number.

Because of the variation between acini it is difficult to be certain whether these changes always occur. Before the age of 4 years, in addition to the above changes numerous alveoli of a new type, so-called 'ductular alveoli' (Boyden, 1967) appear in the wall of pre-acinar bronchioli. They arise as epithelial diverticula which penetrate the peribronchial muscle layer and expand into large alveoli. These probably correspond to the 'spherical alveoli' of the adult (von Hayek, 1960).

Boyden (1967) has described the appearance by the seventh year of the 'accessory bronchiolealveolar' communications of Lambert (1955) which facilitate collateral ventilation. Neither these nor spherical alveoli are sufficient to transform the terminal bronchiolus into a respiratory bronchiolus. A communication resembling a pore of Kohn but between acini has been described at this age (Boyden, 1971). The time of appearance of the pores of Kohn in the human lung has not yet been established but they seem not to be present by 10 months (Marchand, 1912).

At birth, 24 million alveoli are present, and by the age of 8 years this has increased to 300 million (Dunnill, 1962 ; Davies and Reid, 1970) ; according to Weibel (1963) this is the adult number. Recently, Angus and Thurlbeck (1972) have suggested that variation in the adult number is related to height.

Alveolar size changes during growth: in the first three years of life alveolar multiplication keeps pace with increase in lung volume and alveolar size hardly changes. Between 3 and 8 years alveoli increase in size as well as number, thereafter probably only in size (Davies and Reid, 1970). From the age of 4 months the alveolar outline increases in complexity, contributing to the increase in surface area. The area of the air/tissue interface increases from $2.8 \mathrm{~m}^{2}$ at birth to $32 \mathrm{~m}^{2}$ at 8 years, and to $75 \mathrm{~m}^{2}$ in the adult, showing a linear relationship with body surface area (Dunnill, 1962).

A detailed reconstruction of one acinus in a lung of 6 years 8 months has been described by Boyden (1971). By this time the architecture of the acinus is complex, some parts enveloping others. The edges of adjacent acini, and so of lobule and segment, interdigitate like a threedimensional jig-saw puzzle.

Thus, at 7 years (Figure $f$, based on Boyden (1971) ) three generations of respiratory bronchioli are included, six of alveolar ducts, the last ending in an atrium leading into alveolar sacs. From all these structures alveoli of the 'mature' shape arise. The acinus is about $4 \mathrm{~mm}$ long. Since this is the latest age for which an acinus has been reconstructed, it has been chosen as representing the full pattern of development. From this time to adulthood, the pattern probably changes little but airway length and alveolar size increase. In the adult the acinus is $5-10 \mathrm{~mm}$ long.

\section{CONCLUSION}

Most of the airway development within the acinus has occurred by birth. Some airways representing respiratory bronchioli of the adult lung are present by the 19th week of gestation 
and all by 28 weeks' gestation. During childhood one or more generations of respiratory bronchioli may develop proximally by centripetal alveolization of a terminal bronchiolus, but from 16 weeks' gestation to adulthood the maximum number of airway generations thus transformed to respiratory bronchioli is probably only two.

Some of the adult alveolar ducts are first seen as transitional ducts and saccules before the 28th week of gestation: by birth, additional generations of saccules may be present and all are remodelled soon after birth to form alveolar ducts. These may increase in number after birth by division or perhaps by transformation of one generation of respiratory bronchioli. The distal part of the last alveolar duct beyond the level where muscle disappears from the wall develops into the adult atrium. The alveolar sacs and alveoli of the adult do not appear until after birth, the latter increasing in number and size with age.

We are grateful to Professor E. A. Boyden for the instructive and stimulating discussions we have enjoyed with hin during the preparation of this manuscript. To Miss G. Leballeur we are indebted for the diagram.

\section{REFERENCES}

Angus, G. E. and Thurlbeck, W. M. (1972). Number of alveoli in the human lung. Journal of Applied Physiology, 32, 483.

Boyden, E. A. (1965). The terminal air sacs and their blood supply in a 37-day infant lung. American Journal of Anatomy, 116, 413.

- (1967). Notes on the development of the lung in infancy and early childhood. American Journal of Anatomy, 121, 749.

- (1969). The pattern of the terminal air spaces in a premature infant of 30-32 weeks that lived $19 \frac{1}{4} \mathrm{hrs}$. American Journal of Anatomy, 126, 31.

- (1971). The structure of the pulmonary acinus in a child of six years and eight months. American Journal of Anatomy, 132, 275.

- (1972a). Development of the human lung. In Brennemann's Practice of Pediatrics, vol. IV, ch. 64. Harper and Row, Hagerstown.

- (1972b). The structure of compressed lungs in congenital diaphragmatic hernia. American Journal of Anatomy, 134, 497.

- and Tompsett, D. H. (1965). The changing patterns in the developing lungs of infants. Acta Anatomica, 61, 164.
Bucher, U. and Reid, L. (1961). Development of the intrasegmental bronchial tree: the pattern of branching and development of cartilage at various stages of intrauterine life. Thorax, 16, 207.

Campiche, M. A., Gautier, A., Hernandez, E. I., and Reymond, A. (1963). An electron microscope study of the fetal development of human lung. Pediatrics, 32, 976.

Commission on Embryological Terminology (1970). In: Nomina Embryologica, Leningrad, 1970, edited by L. B. Arey and H. W. Mossman. Federation of American Societies for Experimental Biology (FASEB), Bethesda, Maryland.

Davies, G. and Reid, L. (1970). Growth of the alveoli and pulmonary arteries in childhood. Thorax, 25, 669.

Dunnill, M. S. (1962). Postnatal growth of the lung. Thorax, 17, 329.

Elliott, F. M. and Reid, L. (1965). Some new facts about the pulmonary artery and its branching pattern. Clinical Radiology, 16, 193.

von Hayek, H. (1960). The Human Lung. English translation by V. E. Krahl. Hafner Publishing Company, New York.

Hislop, A. and Reid, L. (1972). Intrapulmonary arterial development during fetal life-branching pattern and structure. Journal of Anatomy, 113, 35.

(1973a). Pulmonary arterial development during childhood: branching pattern and structure. Thorax, 28, 129.

(1973b). Fetal and childhood development of the intrapulmonary veins in man-branching pattern and structure. Thorax, 28, 313.

Lambert, M. W. (1955). Accessory bronchiole-alveolar communications. Journal of Pathology and Bacteriology, 70, 311 .

Lauweryns, J. M. and Rosan, R. C. (1971). The unit lobule: a revised concept of the neonatal lung. In Proceedings of the 2nd European Congress on Perinatal Medicine, London 1970. pp. 259-263. ed. by P. J. Huntingford, R. W. Beard, F. E. Hytten, and J. W. Scopes. Karger, Basel.

Loosli, C. G. and Potter, E. L. (1951). The prenatal development of the human lung (abstract). Anatomical Record, 109, 320.

- (1959). Pre- and postnatal development of the respiratory portion of the human lung. American Review of Respiratory Diseases, 80, Suppl. 5.

Marchand, R. (1912). Les pores des alvéoles pulmonaires. Bibliographie Anatomique (Paris), 22, 57.

Meyrick, B. and Reid, L. (1970). The alveolar wall. British Journal of Diseases of the Chest, 64, 121.

Miller, W. S. (1937). The Lung. Thomas, Springfield, Illinois.

Palmer, D. M. (1936). The lung of a human foetus of $170 \mathrm{~mm}$ C.R. length. American Journal of Anatomy, 58, 59.

Reid, L. and Rubino, M. (1959). The connective tissue septa in the foetal human lung. Thorax, 14, 3.

Weibel, E. (1963). Morphometry of the Human Lung. SpringerVerlag, Berlin. 